\title{
Terminologia especializada de enfermagem para cuidado à pessoa com insuficiência cardíaca crônica
}

\author{
Specialized nursing terminology for the care of people with chronic heart failure \\ Terminología de enfermería especializada para el cuidado de \\ personas con insuficiencia cardíaca crónica
}

Maria Naiane Rolim Nascimento ${ }^{1}$ (1) Antonia Elizangela Alves Moreira ${ }^{1}$ (C) Natana de Morais Ramos ${ }^{2}$ (D) Emiliana Bezerra Gomes ${ }^{1}$ (]) Nuno Damácio de Carvalho Félix ${ }^{3}$ (1) Célida Juliana de Oliveira $^{1}$ (D)

1. Universidade Regional do Cariri. Crato, $\mathrm{CE}$, Brasil.

2. Universidade Estadual do Ceará. Fortaleza, CE, Brasil.

3. Universidade Federal do Recôncavo da Bahia. Santo Antonio de Jesus, BA, Brasil.

Autor correspondente:

Maria Naiane Rolim Nascimento.

naianerolim@hotmail.com.

Recebido em 04/08/2020.

Aprovado em 21/10/2020.

DOl:https://doi.org/10.1590/2177-9465-EAN-2020-0306

\section{REsUmo}

Objetivo: Construir uma terminologia especializada de enfermagem para o cuidado à pessoa com insuficiência cardíaca crônica. Método: Estudo metodológico, realizado em duas etapas: identificação dos conceitos relevantes em artigos científicos relacionados à prioridade de saúde eleita e mapeamento cruzado dos conceitos identificados com os conceitos da CIPE ${ }^{\circledR}$. Resultados: Extraíram-se 333 conceitos únicos úteis ao cuidado de enfermagem relacionado à afecção em questão, logo submetidos à etapa do mapeamento cruzado com os conceitos da CIPE ${ }^{\circledR 2019-2020 . ~ F o r a m ~ c l a s s i f i c a d o s ~} 201$ conceitos como constantes e 132 não constantes. Na análise de similaridade e abrangência, $85 \%$ foram classificados como iguais e $15 \%$ similares. Os conceitos não constantes caracterizaram-se $44 \%$ como mais abrangentes, $11 \%$ mais restritos e $45 \%$ sem concordância. Quanto à classificação dos conceitos nos sete Eixos da CIPE ${ }^{\circledR}$, caracterizou-se por Eixo Foco (50\%), Eixo Julgamento (13\%), Eixo Meio (12\%), Eixo Ação (10\%), Eixo Localização (9\%), Eixo Tempo (4\%) e Eixo Cliente (2\%). Conclusões e implicações para a prática: Foi possível construir terminologia especializada de enfermagem com base na CIPE ${ }^{\circledR}$ para o cuidado à pessoa com insuficiência cardíaca crônica, em que $40 \%$ dos conceitos que compõem a terminologia não estão constantes na versão 2019-2020 da classificação, sendo passíveis de inserção em versão posterior.

Palavras-chave: Enfermagem; Terminologia Padronizada em Enfermagem; Classificação; Reabilitação Cardíaca; Insuficiência Cardíaca.

\section{Abstract}

Objective: To build specialized Nursing terminology for the care of people with chronic heart failure. Method: Methodological study, carried out in two stages: identification of relevant concepts in scientific articles related to the elected health priority and cross-mapping of the concepts identified with the ICNP ${ }^{\otimes}$ concepts. Results: A total of 333 unique concepts useful for Nursing care related to the condition in question were extracted, soon submitted to the cross-mapping stage with the concepts of ICNP 2019-2020. 201 concepts were classified as constant and 132 as non-constant. In the analysis of similarity and comprehensiveness, $85 \%$ were classified as equal and $15 \%$ as similar. The non-constant concepts were characterized by $44 \%$ as more comprehensive, $11 \%$ more restricted and $45 \%$ without agreement. As for the classification of concepts in the seven ICNP ${ }^{\circledast} \mathrm{Axes}$, it was characterized by the Focus Axis ( $50 \%$ ), Judgment Axis (13\%), Middle Axis (12\%), Action Axis (10\%), Location Axis ( $9 \%)$, Time Axis $(4 \%)$ and Customer Axis (2\%). Conclusions and contributions to the practice: It was possible to build specialized Nursing terminology based on the ICNP ${ }^{\otimes}$ for the care of people with chronic heart failure, in which $40 \%$ of the concepts that make up the terminology are not included in the 2019-2020 version of the classification, being subjected to insertion in a later version.

Keywords: Nursing; Standardized Nursing Terminology; Classification; Cardiac Rehabilitation; Heart Failure.

\section{Resumen}

Objetivo: Construir una terminología de enfermería especializada para el cuidado de personas con insuficiencia cardíaca crónica. Método: Estudio metodológico, realizado en dos etapas: identificación de conceptos relevantes en artículos científicos relacionados con la prioridad de salud elegida y mapeo cruzado de los conceptos identificados con los conceptos de ICNP . $^{\circ}$ Resultados: Se extrajeron 333 conceptos únicos útiles para la atención de enfermería relacionados con la afección en cuestión que luego se sometieron a la etapa de mapeo cruzado con los conceptos de CIPE ${ }^{\circledR} 2019-2020.201$ conceptos se clasificaron como constantes y 132 como no constantes. En el análisis de similitud y exhaustividad, el $85 \%$ se clasificó como iguales y el 15\%, como similares. Los conceptos no constantes se caracterizaron en un $44 \%$ como más completos, $11 \%$ más restringidos y $45 \%$ sin acuerdo. En cuanto a la clasificación de conceptos en los siete Ejes CIPE ${ }^{\circledast}$, se caracterizó por el eje de Enfoque (50\%), el Eje de Juicio (13\%), el Eje Medio (12\%), el Eje de Acción (10\%), el Eje de Ubicación (9\%), Eje de Tiempo (4\%) y Eje de Cliente (2\%). Conclusiones y contribuciones a la práctica: fue posible construir una terminología de enfermería especializada basada en $\mathrm{CIPE}^{\circledast}$ para el cuidado de personas con insuficiencia cardíaca crónica, en la cual el $40 \%$ de los conceptos que componen la terminología no están incluidos en la versión 2019-2020 de la clasificación, estando sujetos a inserción en una versión posterior.

Palabras clave: Enfermería; Terminología Normalizada de Enfermería; Clasificación; Rehabilitación Cardiaca; Insuficiencia Cardíaca. 


\section{INTRODUÇÃO}

A insuficiência cardíaca crônica configura-se como condição clínica de caráter sistêmico, em que o músculo cardíaco não consegue ejetar sangue suficiente para atender às demandas do metabolismo tissular, compreendendo a etiologia sistólica, diastólica ou ambas e pode resultar de outras comorbidades cardiovasculares prévias. ${ }^{1}$ Estima-se que existam aproximadamente 23 milhões de casos de insuficiência cardíaca em todo o mundo, ${ }^{2}$ com $17 \%$ a $45 \%$ das mortes dentro do primeiro ano após o diagnóstico e a maioria nos primeiros cinco anos após o diagnóstico clínico. ${ }^{3}$

A pessoa com insuficiência cardíaca geralmente é submetida a internações recorrentes pelo fato de não procurar atendimento adequado e oportuno no controle da afecção por meio da reabilitação cardíaca, o que se configura como desafio diário aos profissionais da saúde nos mais variados níveis de atenção e impacta diretamente no aumento das admissões hospitalares. ${ }^{4}$ O controle dessa afecção está pautado na prevenção de processos agudos e consequentes complicações, por meio de assistência interdisciplinar qualificada no controle e reabilitação cardíaca pós-diagnóstico para que se possa retomar a rotina diária com autonomia. ${ }^{5}$

No cotidiano de trabalho a equipe multiprofissional se comunica por meio de conceitos que configuram os fenômenos do domínio de conhecimento da área de atuação, ou seja, à terminologia de especialidade que caracteriza a linguagem própria e particular desses profissionais. ${ }^{6} \mathrm{Na}$ Enfermagem, especificamente, se detêm os fenômenos caracterizados pelos diagnósticos, resultados e intervenções de enfermagem, como necessidades baseadas nos problemas reais e/ou potenciais identificadas nas pessoas a partir da sua situação de saúde e contexto inserido, que devem ser nomeados por meio da utilização de sistemas de linguagem padronizada/sistemas de classificação. ${ }^{7}$

A Classificação Internacional para a Prática de Enfermagem $\left(\mathrm{CIPE}^{\circledR}\right)$ se caracteriza por ser um sistema de classificação detentor de uma linguagem unificada, abrangendo conceitos diagnósticos/resultados e intervenções de Enfermagem como elementos inter-relacionados e interdependentes, além de conceitos primitivos, que possibilitam a construção de enunciados de enfermagem com base no seu contexto de atuação. ${ }^{8}$

Os conceitos primitivos compõem terminologias de Enfermagem, que devem ser abrangentes e compreender aspectos relacionados a determinadas áreas da saúde, enquanto prioridades de saúde ou necessidades de uma população específica. No contexto cardiovascular, destaca-se terminologia produzida para a pessoa com síndrome metabólica e, em outras áreas de especialidade e/ou populações específicas, para pessoa com lesão por pressão, para a pessoa com lesão medular, para a pessoa com hanseníase e para a pessoa com ostomia..$^{9-13}$

Há escassez na literatura quando se trata de uma terminologia específica, padronizada e validada, direcionada ao cuidado de enfermagem à pessoa com insuficiência cardíaca crônica em suas mais variadas vertentes, ${ }^{14}$ assim, este estudo apresenta potencial inovador pelo intuito de contribuir para a geração de indicadores assistenciais de enfermagem para posterior inserção em sistemas de informação em saúde e com possível impacto no cuidado e reabilitação cardíaca da pessoa com insuficiência cardíaca. Deste modo, objetivou-se construir uma terminologia especializada de enfermagem com base na Classificação Internacional para a Prática de Enfermagem para o cuidado à pessoa com insuficiência cardíaca crônica.

\section{MÉTODO}

Estudo metodológico, seguindo as recomendações para construção de terminologias: 1) Identificação dos conceitos relevantes para a prioridade de saúde eleita; 2) Mapeamento cruzado dos conceitos identificados com os conceitos da CIPE ${ }^{\circledR} .^{15}$

Na primeira etapa, realizou-se uma revisão de literatura no mês de agosto de 2020, para identificar os cuidados de enfermagem à pessoa com insuficiência cardíaca. A busca foi realizada de forma independente por duas pesquisadoras, via portal de periódicos da Coordenação de Aperfeiçoamento de Pessoal de Nível Superior (CAPES) nas bases de dados: Cummulative Index to Nursing and Allied Health Literature (CINAHL), Banco de Dados em Enfermagem (BDENF), Literatura Latino-Americana e do Caribe em Ciências da Saúde (LILACS) e Medical Literature Analysis and Retrieval System Online (MEDLINE via EBSCO), além de literatura cinzenta, por meio de uma busca aberta manual com os descritores, dado o interesse por novos achados passíveis de serem utilizados enquanto arcabouço teórico.

A elegibilidade dos estudos se deu com base na aplicação dos critérios de inclusão: artigos originais e estudos/relatos de caso; produzidos por enfermeiro; disponíveis na íntegra; sem delimitação de escopo temporal; nos idiomas português, inglês e espanhol; e o critério de exclusão: artigos que não respondiam à questão de pesquisa e que tratassem do contexto multiprofissional.

Os artigos selecionados foram utilizados como bases empíricas para a extração dos conceitos clínica e culturalmente relevantes para a prática de enfermagem. Eles foram organizados em arquivos individuais no formato Word for Windows ${ }^{\circledR}$ versão 2013, dos quais foram excluídos os títulos, resumos, descritores, aspectos descritivos sobre os periódicos, identificação e credenciais dos autores, referências, quadros e seus títulos e legendas, deixando apenas a parte textual dos artigos, utilizada para a extração dos conceitos.

Os arquivos do Word for Windows ${ }^{\circledR}$ foram convertidos para o formato .PDF e aplicados no programa PORONTO, ferramenta para construção semiautomática de ontologias a partir de textos em português na área de saúde, desenvolvida em tecnologia de código aberto e gratuita, que extrai os conceitos e suas relações taxonômicas, além de fornecer a sua frequência de aparições. ${ }^{16}$ Ressalta-se que os artigos em outros idiomas que não fossem o português foram traduzidos na íntegra para a sua aplicação no PORONTO, devido a ferramenta reconhecer apenas esse idioma. Tal ferramenta tem sido utilizada em estudos com esse mesmo desenho metodológico e tem demonstrado eficiência na extração dos conceitos. ${ }^{13,17}$ 
Após a extração, os conceitos foram alocados em uma planilha no formato Excel for Windows ${ }^{\circledR}$ versão 2013 para a realização do processo de normalização e uniformização com análise e exclusão de sinônimos, adequação dos tempos verbais e do gênero gramatical, de número (singular e plural) e de sigla que identifique determinado conceito. Foram excluídos os conceitos considerados sem nenhuma ligação com o cuidado de enfermagem direcionado às pessoas com insuficiência cardíaca crônica.

$\mathrm{Na}$ segunda etapa, foi realizado o mapeamento cruzado ou cross mapping dos conceitos extraídos com os conceitos do modelo dos sete Eixos da CIPE ${ }^{\circledR}$ 2019-2020, que se caracteriza como procedimento metodológico para comparar conceitos de diferentes versões da classificação por meio do cruzamento de dados e deliberação da equivalência semântica, para solucionar possíveis vieses, possibilitando a adaptação para uma linguagem padronizada. ${ }^{18}$

Foram utilizadas duas planilhas no Excel for Windows ${ }^{\circledR}$ versão 2013, uma com os conceitos únicos padronizados e normalizados previamente e outra com os conceitos da CIPE ${ }^{\circledR}$ 2019-2020, sendo essas planilhas entrecruzadas pelo programa Access for Windows ${ }^{\circledR}$ versão 2013, para identificação dos conceitos constantes e não constantes na atual versão da CIPE ${ }^{\circledR}$. Conforme o mapeamento, os conceitos foram padronizados em relação à grafia e repetições, no intuito de verificar a adequação desses conceitos e os que foram considerados como similares eram designados baseados na Classificação.

Os conceitos que não foram classificados como iguais foram ainda analisados quanto à similaridade e abrangência em relação aos conceitos constantes na $\mathrm{CIPE}{ }^{\circledR} \cdot{ }^{19} \mathrm{~A}$ literatura propõe que similar é quando não existe concordância da grafia, mas o seu significado é idêntico; mais abrangente quando o mesmo tem um significado maior do que o conceito existente na CIPE ${ }^{\circledR}$; mais restrito quando o conceito tem um significado menor do que o existente na CIPE ${ }^{\circledR}$; e não existe concordância quando o conceito é totalmente diferente do conceito existente na CIPE ${ }^{\circledR}$, ou seja, um novo conceito. Essa etapa ainda possibilitou o enquadramento dos conceitos em um dos sete Eixos da CIPE ${ }^{\circledR} .{ }^{20}$

Os resultados foram analisados de forma descritiva, apresentando a frequência absoluta e relativa dos dados. Considerando a natureza da pesquisa metodológica e o não envolvimento de seres humanos, não se fez necessária apreciação do Comitê de Ética em Pesquisa.

\section{RESULTADOS}

Dos 27 artigos selecionados foram extraídos 86.013 conceitos simples e compostos (incluindo as repetições) sendo destes, 15.763 conceitos únicos. Logo após, foram selecionados os conceitos considerados úteis ao cuidado de enfermagem relacionado à afecção em questão, permanecendo 333 conceitos únicos dentre simples e compostos.

A diminuição significativa no número de conceitos justifica-se dado o processo de normalização e uniformização, que levou em consideração aspectos como palavras sinônimas, siglas já descritas, conceitos médicos, doenças e medicações, tempo verbal diferente, erros de digitação e/ou ortográficos, conceitos casuais e palavras com classes gramaticais distintas, porém com o mesmo significado semântico.

O número de aparições dos conceitos variou de uma a 473 vezes. Os conceitos extraídos com frequência de aparição no corpus de análise maior ou igual a 30 em ordem decrescente foram descritos na Tabela 1.

Os conceitos extraídos foram submetidos ao processo de mapeamento cruzado (cross maping) com os conceitos da CIPE ${ }^{\circledR}$ versão 2019-2020, sendo verificados 201 (60\%) conceitos constantes (Quadro 1), analisados quanto à similaridade e abrangência, com 170 (85\%) classificados como iguais aos da CIPE $^{\circledR}$ e 31 (15\%) considerados similares. Destaca-se que os conceitos classificados como iguais aos da CIPE ${ }^{\circledR}$ foram apresentados com o respectivo código de classificação da CIPE ${ }^{\circledR}$.

Dos 333 conceitos, 132 (40\%) foram classificados como não constantes no sistema de classificação (Quadro 2), que se caracterizaram 58 conceitos como mais abrangentes (44\%), 15 como mais restritos (11\%) e 59 como sem concordância (45\%) com os conceitos da CIPE ${ }^{\circledR}$.

Quanto à classificação dos conceitos em um dos sete Eixos da CIPE ${ }^{\circledR}$, a terminologia especializada de enfermagem caracterizou-se por 167 (50\%) conceitos no Eixo Foco, 45 (13\%) no Eixo Julgamento, 39 (12\%) no Eixo Meio, 32 (10\%) no Eixo Ação, 29 (9\%) no Eixo Localização, 14 (4\%) no Eixo Tempo e sete $(2 \%)$ no Eixo Cliente, em que é possível visualizar todos os conceitos constantes e não constantes.

\section{DISCUSSÃO}

A construção de uma terminologia especializada de enfermagem, como passo inicial para subsidiar a construção de um subconjunto terminológico para o cuidado de enfermagem à pessoa com insuficiência cardíaca crônica, vai ao encontro à padronização e universalização da linguagem de Enfermagem, a fim de evidenciar os conceitos utilizados na prática, contribuindo para o avanço científico nesta área, o que contribui com a melhoria da assistência prestada a esta população específica. ${ }^{11}$

São discutidos com destaque na literatura conceitos com frequência de aparição maior ou igual a 30 no corpus de análise, reflexos do seu impacto no cuidado à pessoa com insuficiência cardíaca, como por exemplo, "família", "adesão", "comunicação", "ansiedade", "educativo", "abordagem" e "espiritualidade", apesar de os três últimos não constarem na classificação, o que indica a importância de avaliar a literatura na área em busca de novos conceitos que possam ser úteis ao cuidado.

O conceito "abordagem" é referido por autores como relacionamento entre o binômio pessoa cuidada-profissional que deve preservar a dignidade de cada um, a escuta das necessidades e a inclusão destas no processo de tomada de decisão centrado no indivíduo, fator de importância no cuidado a pessoa com insuficiência cardíaca, com base na sua reabilitação cardíaca pós-diagnóstico clínico e ações individualizadas, conforme necessidades e contexto inserido. ${ }^{20}$ 
Tabela 1. Conceitos com frequência de aparição no corpus de análise maior ou igual a 30, Crato, Brasil, 2020.

\begin{tabular}{|cccccccc}
\hline CONCEITO & F* $^{*}$ & CONCEITO & F* $^{*}$ & CONCEITO & F* $^{*}$ & CONCEITO & F* $^{*}$ \\
\hline Família & 473 & Peso & 124 & Medida & 73 & Autogerenciamento & 43 \\
\hline Hospitalar & 416 & Domiciliar & 121 & Utilizar & 72 & Congestão & 43 \\
\hline Tratamento & 410 & Papel & 121 & Autocuidado & 71 & Dificuldade & 42 \\
\hline Qualidade & 310 & Mudança & 120 & Hora & 69 & Espiritualidade & 42 \\
\hline Educativo & 254 & Medicamentoso & 118 & Exame & 67 & Continuidade & 41 \\
\hline Conhecimento & 251 & Desenvolver & 117 & Fadiga & 67 & Observar & 40 \\
\hline Atividade & 229 & Cardíaco & 113 & Presença & 65 & Líquido & 39 \\
\hline Controle & 222 & Característica & 107 & Autogestão & 62 & Pensamento & 39 \\
\hline Comportamento & 214 & Manejo & 101 & Analisar & 60 & Episódio & 38 \\
\hline Avaliar & 212 & Redução & 101 & Membro & 60 & Esperança & 36 \\
\hline Taxa & 202 & Frequência & 100 & Área & 57 & Nutricional & 36 \\
\hline Depressão & 175 & Dispneia & 98 & Crônico & 57 & Espiritual & 34 \\
\hline Aumentar & 173 & Monitorar & 96 & Comunidade & 56 & Demanda & 33 \\
\hline Sintoma & 172 & Habilidade & 93 & Exercício Físico & 56 & Intolerância & 33 \\
\hline Acompanhar & 171 & Atenção & 91 & Restrição & 54 & Baixo & 32 \\
\hline Estilo & 171 & Resposta & 89 & Aplicar & 50 & Grau & 32 \\
\hline Terapia & 170 & Débito Cardíaco & 88 & Efetivo & 50 & Procedimento & 32 \\
\hline Condição & 165 & Percepção & 87 & Preocupação & 49 & Respiratório & 31 \\
\hline Risco & 154 & Indivíduo & 84 & Comunicação & 48 & Satisfatório & 31 \\
\hline Cuidador & 149 & Abordagem & 82 & Tamanho & 48 & Sono & 30 \\
\hline Adesão & 145 & Ambiente & 78 & Dor & 47 & Terapêutico & 30 \\
\hline Necessidade & 137 & Edema & 77 & Padrão & 46 & & \\
\hline Ansiedade & 136 & Dieta & 74 & Déficit & 45 & 44 & \\
\hline Capacidade & 124 & Melhorado & 74 & Cateter & & \\
\hline Fequar & & & & \\
\hline
\end{tabular}

*F: frequência absoluta

Conceitos como "família" e "educativo" também são evidenciados quando se referem à realização de intervenções educacionais com os entes da família associadas à redução de readmissões dessas pessoas na rede hospitalar, além dos conceitos "comunicação"e "adesão" ao elucidar que estratégias utilizadas para educação em saúde são primordiais, no diz respeito à adesão ao tratamento, quando se insere a família no processo comunicativo, especialmente quando associados a um maior número de consultas de enfermagem prévias. ${ }^{21,22}$

Já o conceito "espiritualidade" foi referido como tema que acarreta efeitos positivos na insuficiência cardíaca, devido ao fato de empoderar e atuar no enfrentamento da afecção, indicada como um resultado positivo, com maior adesão após o diagnóstico da insuficiência cardíaca. ${ }^{23} \mathrm{Faz}-\mathrm{se}$ importante o debate acerca de aspectos que ultrapassem as dimensões biológicas e sociais, como é o caso da espiritual, de forma complementar e interdependente, com a pretensão de compreender a pessoa cuidada como um todo indivisível.
Nos achados verificou-se ainda expressiva quantidade de conceitos constantes na CIPE ${ }^{\circledR}$ 2019-2020, o que explicita que a grande maioria desses referentes à prática clínica de enfermagem à pessoa com insuficiência cardíaca estão contidos na terminologia. Isso demonstra sua utilidade enquanto tecnologia passível de ser utilizada para inserir dados em sistemas eletrônicos de informação em saúde, instrumentos assistenciais e registro da prática clínica de enfermagem em âmbito mundial, contribuindo na geração de indicadores de Enfermagem.

Apesar de apresentar menor prevalência, vale ressaltar o quantitativo de conceitos não constantes na classificação (40\%), o que confere a necessidade de atualização e inserção desses conceitos na $\mathrm{CIPE}^{\circledR}$ com o intuito de preencher lacunas na construção de conceitos pré-coordenados e evidenciar os fenômenos de interesse do domínio da prática de enfermagem para a pessoa com insuficiência cardíaca.

Quanto à classificação dos conceitos por Eixos da $\mathrm{CIPE}^{\circledR}$, destaca-se a prevalência de conceitos pertencentes ao Eixo 
Quadro 1. Conceitos primitivos classificados como constantes na CIPE ${ }^{\circledR}$ 2019-2020, Crato, Brasil, 2020.

\begin{tabular}{|c|c|}
\hline Eixo & Conceitos constantes na CIPE ${ }^{\circledR}$ 2019-2020 \\
\hline Julgamento $(n=22)$ & $\begin{array}{l}\text { Acentuado, Alto (10009007), Aumentado, Baixo (10011438), Cansaço, Dependência (10026671), } \\
\text { Diminuído, Efetivo, Eficaz (10014956), Excessivo, Expandido, Extensão (10007423), Grau (10005663), } \\
\text { Limitado, Melhorado (10026692), Moderado (10025865), Normal (10013295), Pequeno (10018315), } \\
\text { Prejudicado (10012938), Presença (10046624), Risco (10015007), Tamanho (10018218) }\end{array}$ \\
\hline Foco $(n=110)$ & 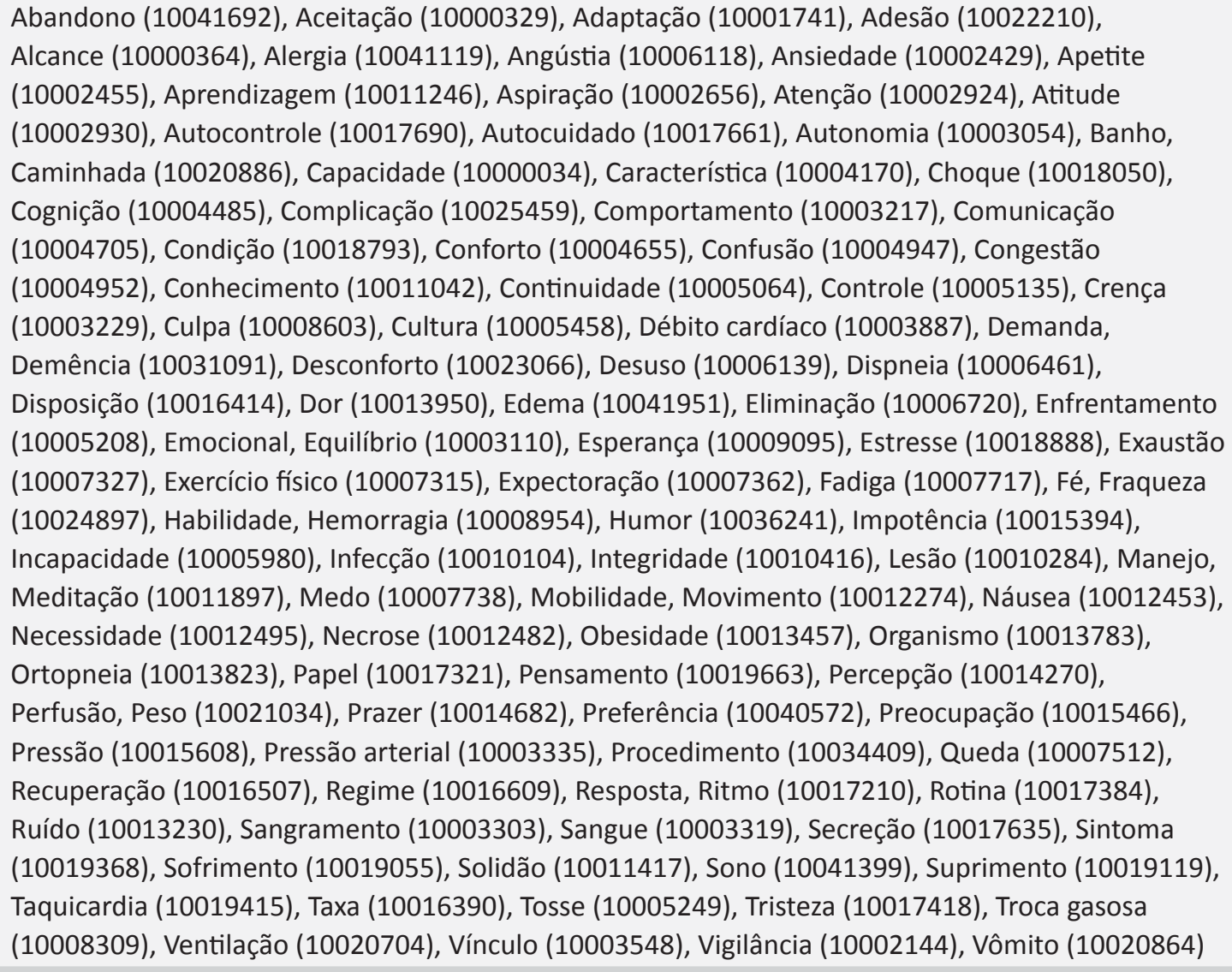 \\
\hline Meio $(n=9)$ & $\begin{array}{l}\text { Analgesia, Atividade física, Cama (10003168), Cateter (10004087), Educação em saúde, Hidratação, } \\
\text { Refeição (10011809), Solução (10018499), Terapia (10019628) }\end{array}$ \\
\hline Localização (n=16) & $\begin{array}{l}\text { Abdominal, Artéria (10002562), Hospitalar, Inferior (10011440), Meio (10012022), Mucosa, Muscular, } \\
\text { Nasal, Oral, Pele (10018239), Periférico (10014386), Pescoço (10012476), Pulmão (10011486), } \\
\text { Superior (10020325), Tórax (10019692), Vascular }\end{array}$ \\
\hline Ação $(n=28)$ & $\begin{array}{l}\text { Acompanhar (10042609), Aconselhar (10005254), Administrar (10001773), Adquirir, Agendar } \\
\text { (10017528), Ajustar (10001760), Aliviar (10002171), Analisar (10002298), Aplicar (10002464), } \\
\text { Apoiar (10019142), Aumentar (10009961), Avaliar (10007066), Cateterizar (10004094), Desenvolver } \\
\text { (10005848), Determinar (10005824), Elevar (10006691), Encorajar (10006823), Fornecer (10015935), } \\
\text { Imobilizar (10009762), Modificar, Monitorar (10012154), Observar (10013474), Obter (10013572), } \\
\text { Preparar (10015478), Prevenir (10015620), Reduzir, Regular (10016613), Verificar (10011813) }\end{array}$ \\
\hline Tempo $(n=11)$ & $\begin{array}{l}\text { Agudo (10001739), Contínuo (10005086), Crônico (10004395), Exame (10007241), Episódio } \\
\text { (10007239), Frequência (10008234), Início (10013689), Manhã (10012226), Noite (10013207), } \\
\text { Noturno, Semana (10021010) }\end{array}$ \\
\hline Cliente $(n=5)$ & $\begin{array}{l}\text { Adulto (10001889), Comunidade (10004733), Cuidador (10003958), Família (10007554), Indivíduo } \\
\text { (10010018) }\end{array}$ \\
\hline
\end{tabular}

${ }^{*}$ n: valor absoluto. Fonte: Dados da pesquisa e $\mathrm{CIPE}^{\circledR}$. 
Quadro 2. Conceitos primitivos classificados como não constantes na CIPE ${ }^{\circledR}$ 2019-2020, Crato-Brasil, 2020.

\begin{tabular}{|c|c|}
\hline Eixo & Conceitos não constantes na $\mathrm{CIPE}^{\circledR}$ \\
\hline Julgamento $(n=23)$ & $\begin{array}{l}\text { Adventício, Antropométrico, Aperto, Ausente, Calórico, Conflitante, Depressivo, Descompensado, } \\
\text { Diastólico, Espiritual, Farmacológico, Glicêmico, Inadequado, Ineficaz, Negativo, Positivo, Paroxístico, } \\
\text { Perturbado, Psicológico, Psicossocial, Sistólico, Satisfatório, Tranquilo }\end{array}$ \\
\hline Foco $(n=57)$ & $\begin{array}{l}\text { Abordagem, Acuidade, Angina, Atividade, Autogerenciamento, Autogestão, Balanço de fluido, Bulha } \\
\text { cardíaca, Circunferência, Colesterol, Comprometimento, Conduta, Convívio, Crepitação, Déficit, } \\
\text { Deambulação, Depressão, Dieta, Dificuldade, Dimensão, Distensão de veia, Distúrbio, Enchimento, } \\
\text { Espiritualidade, Estilo, Estímulo, Fator de risco, Fumo, Hábito, Higiene, Inapetência, Índice de massa } \\
\text { corpórea, Ingesta, Isolamento, Intolerância, Irritação, Mente, Murmúrio, Nutrição, Oferta, Padrão, Paz, } \\
\text { Pulso, Qualidade, Religião, Religioso, Restrição, Retenção, Respeito, Sensação, Sensibilidade, Sentimento, } \\
\text { Tabagismo, Teor, Urgência, Vertigem, Volume }\end{array}$ \\
\hline Meio $(n=30)$ & $\begin{array}{l}\text { Automedicação, Autorrelato, Biopsicossocial, Decúbito, Diurese, Educativo, Excreção, Glicemia, Hídrico, } \\
\text { Líquido, Medicamentoso, Motor, Operatório, Oxigênio, Oximetria, Promoção da saúde, Reflexão, } \\
\text { Relaxamento, Repouso, Sensorial, Sexual, Social, Roupa, Seguro, Medida, Oração, Resíduo, Ronco, } \\
\text { Terapêutico, Via }\end{array}$ \\
\hline Localização (n=13) & $\begin{array}{l}\text { Ambiente, Área, Cardíaco, Corporal, Domiciliar, Extremidade, Igreja, Jugular, Membro, Quadrante, } \\
\text { Respiratório, Tecido, Urinário }\end{array}$ \\
\hline Ação $(n=4)$ & Capacitar, Mensurar, Mudar, Utilizar \\
\hline Tempo $(n=3)$ & Diário, Hora, Cotidiano \\
\hline Cliente $(n=2)$ & Companheiro, Cônjuge \\
\hline
\end{tabular}

${ }^{*}$ n: valor absoluto. Fonte: Dados da pesquisa.

Foco da classificação (50\%), como Exercício Físico, Fadiga, Dispneia, o que demonstra potencialidade para a construção de conceitos coordenados, no que se refere a diagnósticos e resultados de enfermagem. Outras terminologias de enfermagem também referem maior prevalência de conceitos do Eixo Foco. 9,11

Ainda quanto à prevalência de conceitos com base nos Eixos, destacaram-se o Eixo Ação com relação aos conceitos constantes e o Eixo Julgamento com relação aos não constantes, sendo esses conceitos primitivos essenciais na construção de conceitos pré-coordenados de intervenções e diagnósticos/ resultados de enfermagem, respectivamente.

Os conceitos exercício físico, fadiga e dispneia são discutidos com base na evidência de que o primeiro se caracteriza como prática que geralmente causa intolerância na pessoa com insuficiência cardíaca, apresentando sintomas como fadiga e dispneia, ou seja, os conceitos se apresentam como primitivos, porém estão relacionados no contexto das pessoas com insuficiência cardíaca. ${ }^{24}$

Nesse sentido, deve-se avaliar a tolerância ou não à atividade física, sempre levando em consideração os estágios da afecção na pessoa cuidada, com base em uma avaliação interdisciplinar e não realizada apenas pela Enfermagem. Assim, a dispneia é referida como causada por esforços, pelo posicionamento (decúbito), além de ser mencionada como sintoma emocional desencadeado pela ansiedade e nervosismo, prejudicando a qualidade de vida. ${ }^{25}$ Destaca-se a ansiedade enquanto conceito do Eixo Foco, também presente na terminologia.

Assim, denota-se a inter-relação entre os mais variados conceitos presentes no cotidiano de cuidado da pessoa com insuficiência cardíaca, revelando linguagem comum ao contexto, o que necessita de etapa essencial na sua construção. O mapeamento cruzado dos conceitos não constantes com os da CIPE ${ }^{\circledR}$ 2019-2020 é um processo essencial que auxilia na consolidação do cuidado de enfermagem, por meio da uniformização da linguagem, documentação de informações, geração de indicadores sensíveis à prática de Enfermagem e, ainda, facilita a comunicação entre os enfermeiros e os demais profissionais de saúde. ${ }^{26}$

Tal processo possibilita verificar a semelhança entre os enunciados presentes nos mais variados sistemas de classificação em enfermagem, necessidades não contempladas ou incompletas e, a partir desta análise, atuar na consolidação da linguagem. ${ }^{27}$ Destacam-se estudos que vêm sendo desenvolvidos nesse contexto, enquanto tendência atual da Enfermagem, indispensável no processo de construção e compreensão de seus fenômenos..$^{14,26}$

A contribuição do presente estudo está pautada no preenchimento de lacuna no conhecimento acerca da construção de terminologia especializada, enquanto instrumento com potencialidade para o desenvolvimento de conceitos diagnósticos, resultados e intervenções de Enfermagem e sua aplicabilidade na prática para os enfermeiros, enquanto aspectos essenciais ao cuidado de Enfermagem à pessoa com insuficiência cardíaca crônica na prática clínica.

\section{CONCLUSÕES E IMPLICAÇÕES PARA A PRÁTICA}

Foi possível construir uma terminologia especializada de enfermagem com base na CIPE ${ }^{\circledR}$ para o cuidado à pessoa com 
insuficiência cardíaca crônica, em que $40 \%$ dos conceitos não estão constantes na versão 2019-2020 da classificação, com considerável quantitativo de conceitos classificados no Eixo Foco. A construção dessa terminologia implica no processo de consolidação da linguagem de Enfermagem e do seu corpo de conhecimentos, no que se refere ao cuidado à pessoa com insuficiência cardíaca, com potencialidade de aplicação na práxis, no que concerne à construção de instrumentos de cuidado como subconjuntos terminológicos e/ou protocolos.

Enquanto limitação deste estudo, destaca-se a extração de conceitos primitivos apenas da literatura na área caracterizando-se enquanto possibilidade de não refletir todas as particularidades do cuidado de enfermagem direcionado a pessoa com insuficiência cardíaca, porém, tal aspecto não prejudicou a qualidade do estudo, que refletiu convergências com a literatura na área, em relação aos conceitos direcionados a prioridade de saúde especificamente.

Como etapas futuras, para a continuidade e colaboração na consolidação da linguagem de enfermagem à pessoa com insuficiência cardíaca, pretende-se a realização de estudos para a construção de diagnósticos, resultados e intervenções de enfermagem e estruturação de subconjunto terminológico da $\mathrm{CIPE}{ }^{\circledR}$ para o cuidado de enfermagem a referida prioridade de saúde.

\section{CONTRIBUIÇÕES DOS AUTORES}

Desenho do estudo: Maria Naiane Rolim Nascimento, Célida Juliana de Oliveira e Nuno Damácio de Carvalho Félix

Aquisição de dados: Maria Naiane Rolim Nascimento

Análise de dados: Maria Naiane Rolim Nascimento, Antonia

Elizangela Alves Moreira e Natana de Morais Ramos

Interpretação dos resultados: Maria Naiane Rolim Nascimento, Nuno Damácio de Carvalho Félix, Emiliana Bezerra Gomes e Célida Juliana de Oliveira

Redação e revisão crítica do manuscrito: Maria Naiane Rolim Nascimento, Antonia Elizangela Alves Moreira, Natana de Morais Ramos, Emiliana Bezerra Gomes, Nuno Damácio de Carvalho Félix e Célida Juliana de Oliveira

Aprovação da versão final do artigo: Maria Naiane Rolim Nascimento, Antonia Elizangela Alves Moreira, Natana de Morais Ramos, Emiliana Bezerra Gomes, Nuno Damácio de Carvalho Félix e Célida Juliana de Oliveira

Responsabilidade por todos os aspectos do conteúdo e a integridade do artigo publicado: Maria Naiane Rolim Nascimento, Antonia Elizangela Alves Moreira, Natana de Morais Ramos, Emiliana Bezerra Gomes, Nuno Damácio de Carvalho Félix e Célida Juliana de Oliveira

\section{EDITOR ASSOCIADO}

Martha Sauthier

\section{REFERÊNCIAS}

1. Rohde LEP, Montera MW, Bocchi EA, Clausell NO, Albuquerque DC Rassi $S$ et al. Sociedade brasileira de cardiologia. diretriz brasileira de insuficiência cardíaca crônica e aguda. Arq Bras Cardiol. 2018;111: http://dx.doi.org/10.5935/abc.20180190.

2. Writing Group Members, Mozaffarian D, Benjamin EJ, Go AS, Arnett DK Blaha MJ, et al. Heart disease and stroke statistics-2016 update: A report from the American Heart Association. Circulation. 2016;133(4):e38-360 http://dx.doi.org/10.1161/CIR.0000000000000350.

3. Ponikowski P, Voors AA, Anker SD, Bueno H, Cleland JGF, Coats AJS et al.2016 ESC guidelines for the diagnosis and treatment of acute and chronic heart failure: the Task Force for the diagnosis and treatment of acute and chronic heart failure of the European Society of Cardiology (ESC): developed with the special contribution of the Heart Failure Association (HFA) of the ESC. Eur J Heart Fail. 2016;37(27):2129-200. http://dx.doi.org/10.1093/eurheartj/ehw128.

4. Taniguchi C, Okada A, Seto N, Shimizu Y. How visiting nurses detec symptoms of disease progression in patients with chronic heart failure. Int J Qual Stud Health Well-being. 2020;15(1):1735768. http://dx.doi. org/10.1080/17482631.2020.1735768.

5. Souza TCTOA, Correia DMS, Nascimento DC, et al. The difficult daily life of heart failure bearing patients. J Res: Fundam Care. 2019;11(5):1340 1346. http://dx.doi.org/10.9789/2175-5361.2019.v1115.1340-1346

6. Garcia TR. Professional language and nursing domain. Texto Contexto Enferm. 2019;28:e20190102. http://dx.doi.org/10.1590/1980-265xtce-2019-0001-0002.

7. Morais SCRV, Nóbrega MML, Carvalho EC. Cross-mapping of results and Nursing Interventions: contribution to the practice. Rev Bras Enferm. 2018;71(4):1883-90. http://dx.doi.org/10.1590/0034-7167-2017-0324.

8. Tosin MHS, Mecone CAC, Oliveira BGRB. International Classification for Nursing Practice - ICNP ${ }^{\circledR}$ : Application to the Brazilian reality. Rev Bras Enferm. 2015;68(4):730-1. http://dx.doi.org/10.1590/00347167.2015680422i.

9. Félix NDC, Nascimento MNR, Ramos NM, Oliveira CJ, Nóbrega MML. Specialized nursing terminology for the care of people with metabolic syndrome. Esc Anna Nery. 2020;24(3):e20190345. http://dx.doi. org/10.1590/2177-9465-EAN-2019-0345.

10. Duarte FHS, Santos WN, Silva FS, Lima DM, Fernandes SF, Silva RAR Terms of specialized nursing language for people with pressure injury. Rev Bras Enferm. 2019;72(4):1028-35. http://dx.doi.org/10.1590/00347167-2018-0492.

11. Clares JWB, Fernandes BKC, Guedes MVC, Freitas MC. Specialized nursing terminology for the care of people with spinal cord injury. Rev Esc Enferm USP. 2019;53:e03445. http://dx.doi.org/10.1590/s1980220x2018014203445.

12. Oliveira MDS, Roque e Lima JO, Garcia TR, Bachion MM. Useful terms for nursing practice in the care of people with leprosy. Rev Bras Enferm. 2019;72(3):744-52. http://dx.doi.org/10.1590/0034-7167-2017-0684.

13. Carvalho CMG, Cubas MR, Nóbrega MML. Terms of the specialized nursing language for the care of ostomates. Rev Bras Enferm. 2017;70(3):461-7. http://dx.doi.org/10.1590/0034-7167-2015-0058.

14. Nascimento MNR, Silva MY, Viana MCA, Oliveira CJ, Martins AKL, Félix NDC. Nursing diagnoses for people with heart failure: cross mapping. Rev enferm UFPE on line. 2019;13:1-8. http://dx.doi.org/10.5205/1981 8963.2019.240194.

15. Nóbrega MML, Cubas MR, Egry EY, Nogueira LGF, Carvalho CMG Albuquerque LM. Desenvolvimento de subconjuntos terminológicos da CIPE ${ }^{\circledast}$ no Brasil. In: Cubas MR, Nóbrega MML. Atenção primária em saúde: diagnóstico, resultado e intervenções de enfermagem. Rio de Janeiro: Elsevier; 2015. p. 25-36.

16. Zahfra FM, Carvalho DR, Malucelli A. Poronto: tool for semi-automatic ontology construction in portuguese. J Health Inform [Internet]. 2013 [citado 2018 abr 12];5(2):52-9. Disponível em: http://www.jhi-sbis.saude. ws/ojs-jhi/index.php/jhi-sbis/article/view/232/167

17. Félix NDC, Ramos NM, Nascimento MNR, Moreira TMM, Oliveira CJ. Nursing diagnoses from ICNP ${ }^{\circledast}$ for people with metabolic syndrome. Rev Bras Enferm.2018;71(Suppl 1):467-74. http://dx.doi.org/10.1590/00347167-2017-0125.

18. International Organization for Standardization. ISO 12300: health informatics: principles of mapping between terminological systems. Genebra: ISO; 2016. p. 1-46. 
19. Nóbrega MML, Cubas MR, Egry EY, Nogueira LGF, Carvalho CMG, Albuquerque LM. Desenvolvimento de subconjuntos terminológicos da CIPE ${ }^{\circledast}$ no Brasil. In: Cubas MR, Nóbrega MML. Atenção Primária em Saúde: diagnósticos, resultados e intervenções de enfermagem. Rio de Janeiro: Elsevier; 2015. p. 3-14.

20. Sampaio C, Renaud I, Leão PP. "The roller coaster ride of heart failure": Nursing staff's perceptions of dignity. Acta Paul Enferm. 2020;33:1-8. http://dx.doi.org/10.37689/acta-ape/2020AO0165.

21. Agarwal KS, Bhimaraj A, Xu J, Bionat S, Pudlo M, Miranda D et al. Decreasing heart failure readmissions among older patients with cognitive impairment by engaging caregivers. J Cardiovasc Nurs. 2020;35(3):253-61. http://dx.doi.org/10.1097/JCN.0000000000000670.

22. Silva AF, Cavalcanti ACD, Malta M, Arruda CS, Gandin T, Fé A et al. Treatment adherence in heart failure patients followed up by nurses in two specialized clinics. Rev. Latino-Am. Enfermagem. 2015;23(5):88894. http://dx.doi.org/10.1590/0104-1169.0268.2628.

23. Moshki M, Khajavi A, Minaee S, Vakilian F, Hashemizadeh H. Perceived benefits of the disease: A qualitative study of patients' experiences of heart failure. Nurs Health Sci. 2020;22(2):464-71. http://dx.doi. org $/ 10.1111 /$ nhs. 12682 .
24. Padua BLR, Vieira GCA, Pereira JMV, Figueiredo LS, Flores PVP, Cavalcanti ACD. Nursing diagnoses activity intolerance in patients with chronic heart failure. Nursing. 2019 [citado 2019 nov 09];22(250):27162720. Disponível em: http://www.revistanursing.com.br/revistas/250/ pg12.pdf

25. Souza TCTOA, Correia DMS, Nascimento DC, Christovam BP, Batista DCS, Cavalcanti ACD. The Difficult Daily Life of Heart Failure Bearing Patients El difícil cotidiano de los pacientes com insuficiencia cardíaca J Res: Fundam Care. online. 2019;11(5):1340-1346. http://dx.doi. org/10.9789/2175-5361.2019.v11i5.1340-1346

26. Silva DVA, Sousa INM, Rodrigues CAO, Pereira FAF, Gusmão ROM, Araujo DD. Nursing diagnoses in a home-based program: cross-mapping and NANDA-I Taxonomy. Rev Bras Enferm. 2019;72(3):584-91. http:// dx.doi.org/10.1590/0034-7167-2018-0323.

27. Tannure MC, Salgado PO, Chianca TCM. Cross-Mapping: Diagnostic labels formulated according to the ICNP ${ }^{\circledR}$ versus diagnosis of NANDA International. Rev Bras Enferm. 2014;67(6):972-8. http://dx.doi. org/10.1590/0034-7167.2014670616. 